\title{
Relevance of magnetic properties of soil in the magnetic observatories to geomagnetic observation
}

\author{
Toshiaki Mishima ${ }^{1}$, Takeshi Owada ${ }^{2}$, Takashi Moriyama ${ }^{2}$, Norihisa Ishida ${ }^{3}$, Kosuke Takahashi $^{2}$, \\ Shingo Nagamachi ${ }^{2}$, Yuki Yoshitake ${ }^{2}$, Yasuhiro Minamoto ${ }^{2}$, Fujio Muromatsu ${ }^{2}$, and Shuichi Toyodome ${ }^{2}$ \\ ${ }^{1}$ Graduate School of Science, Osaka City University, 3-3-138 Sugimoto, Sumiyoshi-ku, Osaka 558-8585, Japan \\ ${ }^{2}$ Kakioka Magnetic Observatory, Japan Meteorological Agency, 595 Kakioka, Ishioka-shi, Ibaraki 315-0116, Japan \\ ${ }^{3}$ Abashiri Local Meteorological Observatory, Japan Meteorological Agency, 2-1-16 Daimachi, Abashiri-shi, Hokkaido 093-0031, Japan
}

(Received May 14, 2012; Revised September 16, 2012; Accepted September 20, 2012; Online published May 7, 2013)

\begin{abstract}
Annual geomagnetic variations with a maximum amplitude of $5 \mathrm{nT}$, and in phase with ground temperature variations at a depth of 1-2 m, were observed in the baseline values of fluxgate magnetometers installed at three JMA magnetic observatories. A possible origin of the annual variations is a change in magnetization of the soil due to changes in ground temperature. In order to examine the effect of temperature changes on soil magnetization, we measured the magnetic properties of soil samples collected at the JMA observatories. Magnetization of soil samples in a magnetic field of $0.05 \mathrm{mT}$ ranged within $0.05 \times 10^{-3}-1.6 \times 10^{-3} \mathrm{~A} \mathrm{~m}^{2} / \mathrm{kg}$ and the temperature dependence of magnetization ranged within $0.3 \times 10^{-6}-14 \times 10^{-6} \mathrm{~A} \mathrm{~m}^{2} / \mathrm{kg}{ }^{\circ} \mathrm{C}$, except for a sample having an extraordinarily strong magnetization. Based on the measured magnetization, and their temperature dependence, of samples from Memambetsu, which shows the largest values among the samples from the three observatories, we determined the distribution of the geomagnetic field and its annual variation produced by soil magnetization. The maximum amplitude of annual variation in the geomagnetic field is $7 \mathrm{nT}$, which is consistent with the observed annual variation of the baseline value of the magnetometers.
\end{abstract}

Key words: Geomagnetic observation, annual variation, rock magnetism.

\section{Introduction}

Geomagnetic observatories record variations of the geomagnetic field with variometers and determine the absolute values of the records with a separate procedure called absolute measurements. Absolute measurements of the total force, inclination and declination of the geomagnetic field are carried out with proton magnetometers and magnetic theodolites. They are converted into the absolute values of the three components of the geomagnetic field at the variometer sensor location, assuming that the difference in the geomagnetic field between the variometer and the absolute measurement sites do not vary with time. The baseline values of the variometers are then calculated as the differences between the variometer records and the absolute values. The magnetic observatories operated by the Japan Meteorological Agency (JMA) (Fig. 1) employ three-axes fluxgate magnetometers as the main variometers (Oowada et al., 2003) and obtain their baseline values by absolute measurements once a week.

The baseline values for the fluxgate magnetometers (96FM, 90FM and 95FM) at Memambetsu (MMB), Kakioka (KAK), and Kanoya (KNY), magnetic observatories (Fig. 2) show different annual variations. At MMB, the amplitude of the annual variation in $Z$ (vertical), and $H$

Copyright (c) The Society of Geomagnetism and Earth, Planetary and Space Sciences (SGEPSS); The Seismological Society of Japan; The Volcanological Society of Japan; The Geodetic Society of Japan; The Japanese Society for Planetary Sciences; TERRAPUB.

doi:10.5047/eps.2012.09.008 (horizontal), components is $5 \mathrm{nT}$ and $2 \mathrm{nT}$, and the $D$ (declination) component does not show any significant annual variation. At $\mathrm{KAK}$, the $H$ and $Z$ components have annual variation amplitudes of $2 \mathrm{nT}$, while the $D$ component does not show any significant annual variation. At KNY, linearly decreasing, and increasing, trends at rates of $1-2 \mathrm{nT} / \mathrm{yr}$ are notable in the $H$ and $Z$ components, and an additional annual variation of amplitude $1 \mathrm{nT}$ is notable in the $H, Z$ and $D$ components.

Observing conditions, such as a change in temperature and the tilt of the magnetometers, are the most plausible causes of annual variations, but cannot fully explain the observed annual variations. The annual variations shown in Fig. 2 are already corrected using tiltmeters installed in the sensors of the magnetometers, and are out of phase with the temperature variation of the magnetometers. Furthermore, differences in total geomagnetic intensities, obtained with three proton magnetometers installed at different locations within MMB, show annual variations with amplitudes of $\sim 1$ nT (Nishimura et al., 2012). As observations with proton magnetometers are not likely to be largely affected by observing conditions, the observed annual variations can be considered to be actual geomagnetic variations.

A possible origin of annual variations is a change in the magnetization of the soil due to changes in the ground temperature. In volcanic areas, the conclusion that geomagnetic annual variations are caused by a change in the magnetization of surface rock produced by a change in ground temperature (Utada et al., 2000), is widely accepted. This 


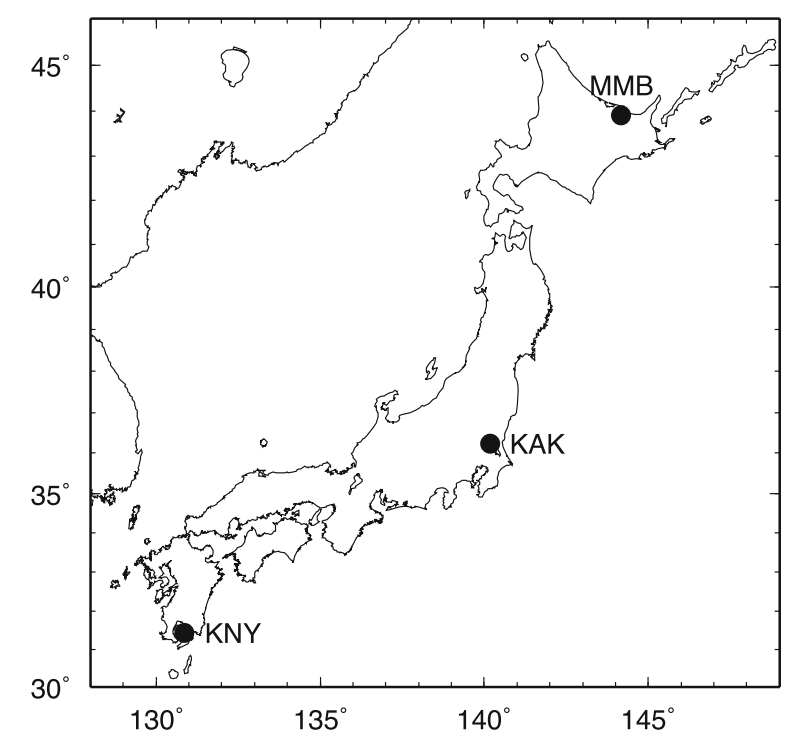

Fig. 1. Geomagnetic observatories operated by JMA. MMB: Memambetsu; KAK: Kakioka; KNY: Kanoya.

MMB

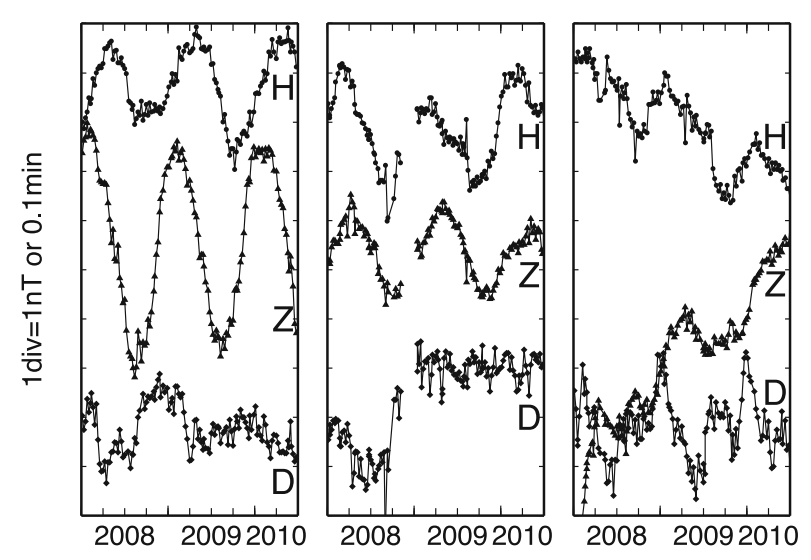

Fig. 2. Baseline values of fluxgate magnetometers installed at Memambetsu, Kakioka and Kanoya Observatories from January 2008 to June 2010.

idea is also applicable to non-volcanic areas in Japan, because surface soil in Japan often contains materials of a volcanic origin. For example, many volcanic tephra layers are widespread within sediments in Hokkaido (Machida and Arai, 2003) and some of them show strong magnetic susceptibility (Kawamura et al., 2007). The change in soil magnetization produced by a change in the ground temperature has also been suggested as a possible cause of the change in geomagnetic observational data at observatories in Japan (e.g., Ogawa and Koyama, 2009; Yamazaki et al., 2012). However, the non-availability of soil magnetic properties has prevented a quantitative evaluation of geomagnetic changes resulting from a temperature change of the soil.

In this study, we measured the magnetic properties of soil samples collected from the three JMA observatories, and we have examined the effect of temperature change on the soil magnetization to the geomagnetic annual variations.

\section{Samples and Methods}

We dug two pits with depths of 1-1.5 m within each observatory, and we collected 1-3 unoriented soil samples at separate depths inside each pit (Fig. 3, Table 1). The sampling depths of each pit were determined based on visual features, such as soil color and grain size, as well as the lithological description of the existing borehole columns drilled within the observatories. We also used a previouslycollected sandy soil sample with strong magnetization (presumably volcanic ash) from the MMB observatory, which was found at a depth of $4 \mathrm{~m}$ when the continuous observation house was built.

The change of magnetization in response to temperature change was measured. The measurement was performed with a Quantum Design Magnetic Property Measurement System (MPMS-XL) at the Geological Survey of Japan, the National Institute of Advanced Industrial Science and Technology. A small subsample ( $100 \mathrm{mg}$ ) of each sample was analyzed. In order to reduce the residual magnetic field in the instrument, the superconducting magnet was quenched before the measurement runs. The applied static magnetic field on a subsample was set to $50000 \mathrm{nT}(0.05 \mathrm{mT})$ in order to impart induced and remanent magnetization. Each subsample was then heated from $2^{\circ} \mathrm{C}$ to $27^{\circ} \mathrm{C}$, at a rate of $1^{\circ} \mathrm{C} / \mathrm{min}$, and the change of magnetization was monitored at intervals of $1^{\circ} \mathrm{C}$.

Low-field magnetic susceptibility was measured with a Bartington MS2B magnetic susceptibility meter at an operating frequency of $465 \mathrm{~Hz}$, a peak applied field of $0.25 \mathrm{mT}$ and a temperature of $26^{\circ} \mathrm{C}$. Subsamples filled into $6.86-\mathrm{cm}^{3}$ paleomagnetic cubes were used for the low-field magnetic susceptibility measurement. Bulk density was calculated from the weight and volume of the subsamples. Low-field magnetic susceptibility and bulk density of the M5 sample were not measured, because the amount of sample available was insufficient.

\section{Results}

Figure 4 shows the change of magnetization in response to temperature change. The magnetization of most samples showed a linear decrease with increasing temperature. A notable change in the decrease rate, between 11 and $19^{\circ} \mathrm{C}$, was shown in the demagnetization curve of the M2 sample. The M5 sample showed a large fluctuation in the demagnetization curve and high standard deviation values in the some measurement data, possibly because the grains in the sample were coarse and loose, and slightly moved during the measurement. We calculated a magnetization value at $12^{\circ} \mathrm{C}$ and the temperature dependence of magnetization with their standard deviations by a linear approximation of these curves (Table 2).

Samples from KNY have the smallest decrease rate $\left(0.3 \times 10^{-6}-1.4 \times 10^{-6} \mathrm{~A} \mathrm{~m}^{2} / \mathrm{kg}^{\circ} \mathrm{C}\right)$ of the three observatories, though the magnetization $\left(0.6 \times 10^{-3}-0.9 \times 10^{-3} \mathrm{~A}\right.$ $\mathrm{m}^{2} / \mathrm{kg}$ ) is not so small. Spatial differences in magnetization and decrease rate were notable between samples from two pits at KAK (K1, K2 and K3, K4). Samples from MMB showed the largest magnetization $\left(0.7 \times 10^{-3}-1.5 \times 10^{-3} \mathrm{~A}\right.$ $\left.\mathrm{m}^{2} / \mathrm{kg}\right)$ and the largest decrease rate $\left(1.7 \times 10^{-6}-14 \times 10^{-6} \mathrm{~A}\right.$ $\left.\mathrm{m}^{2} / \mathrm{kg}{ }^{\circ} \mathrm{C}\right)$, even excluding the M5 sample $\left(4.3 \times 10^{-3} \mathrm{~A}\right.$ 


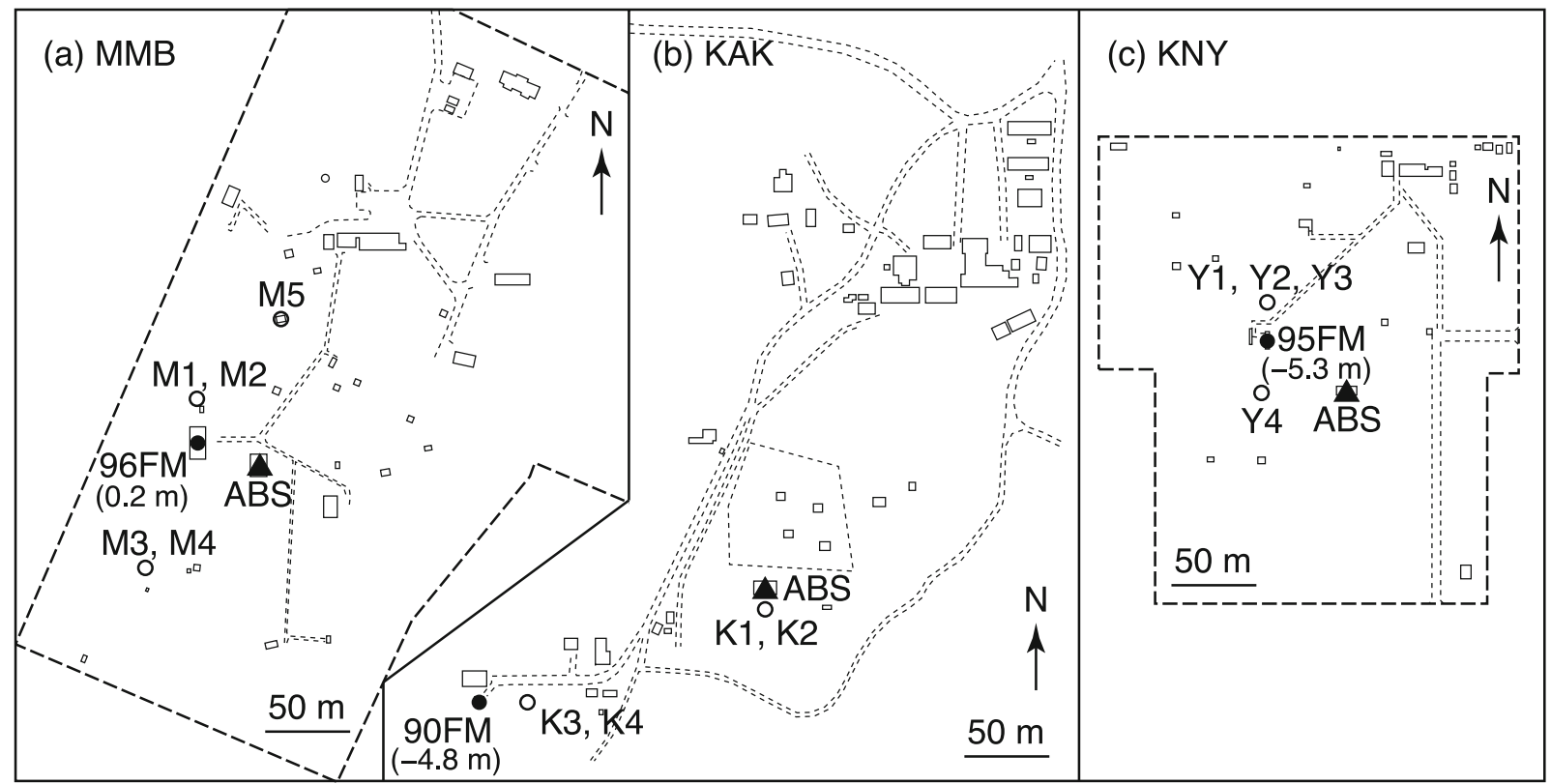

Fig. 3. The observation and sampling locations in (a) Memambetsu, (b) Kakioka and (c) Kanoya Observatories. Solid circles indicate the locations and sensor height of the main fluxgate magnetometers. Solid triangles show the absolute measurement houses. Open circles show the locations of the soil samples.

Table 1. Summary of the collected soil samples from the JMA observatories. Color taken from Oyama and Takehara (1967).

\begin{tabular}{clcl}
\hline Sample & \multicolumn{1}{c}{ Location } & Depth & \\
\hline M1 & MMB North of Variation House \#2 & 0.5 & brown \\
M2 & & 1.1 & yellowish brown \\
M3 & MMB North of Total Forth (79F) & 0.5 & dark brown \\
M4 & & 1.0 & yellowish brown \\
M5 & MMB Continuous Observation House & 4.0 & mixture of transparent, grayish white and black \\
\hline K1 & KAK South of Comparison \& Calibration House & 0.5 & yellowish brown \\
K2 & & 1.0 & olive brown \\
K3 & KAK East of Underground Variation House & 0.5 & dark brown \\
K4 & & 1.0 & dark brown \\
\hline Y1 & KNY North of Underground Variation House & 0.3 & reddish black \\
Y2 & & 1.2 & brown \\
Y3 & & 1.5 & black \\
Y4 & KNY North of Continuous Observation House & 1.4 & mixture of reddish brown and reddish black \\
\hline
\end{tabular}

$\left.\mathrm{m}^{2} / \mathrm{kg} ; 57 \times 10^{-6} \mathrm{~A} \mathrm{~m}^{2} / \mathrm{kg}^{\circ} \mathrm{C}\right)$.

The bulk density of the subsamples filled in the paleomagnetic cubes ranges between $0.87-1.63 \times 10^{3} \mathrm{~kg} / \mathrm{m}^{3}$ (Table 2). The variation in bulk density might be affected by compaction and the void space of the subsamples in the cubes. For convenience, we adopted $1 \times 10^{3} \mathrm{~kg} / \mathrm{m}^{3}$ as the bulk density in order to convert the unit of magnetization.

Low-field magnetic susceptibility ranged from 39.9$848 \times 10^{-5}$ SI units (Table 2). Four samples from MMB, and two samples from KAK (K3 and K4), which have a large magnetization, show high magnetic susceptibility (569$\left.848 \times 10^{-5} \mathrm{SI}\right)$. The samples from KNY show a moderatelyhigh magnetic susceptibility $\left(216-689 \times 10^{-5} \mathrm{SI}\right)$. Two samples from KAK (K1 and K2), which have the smallest magnetization, show the lowest magnetic susceptibility $\left(39.9-73.4 \times 10^{-5} \mathrm{SI}\right)$.

The magnetic susceptibility range of $39.9-848 \times 10^{-5} \mathrm{SI}$ corresponds to an induced magnetization in the range
0.016-0.34 A/m in a AC magnetic field of $50000 \mathrm{nT}$, and is almost a third of the magnetization measured with the MPMS. A possible cause of the difference between the MPMS-measured magnetization and the calculated induced magnetization from the magnetic susceptibility might be a residual magnetic field in the sample position in the MPMS. Although efforts were made to minimize the residual field by using magnet reset, a residual field of $0.1 \mathrm{mT}$ might be possible in an MPMS, which is usually operated with a strong magnetic field up to $5 \mathrm{~T}$. Another possible cause of the difference in magnetization is the viscous remanent behavior of magnetic minerals in the soil. Viscous remanent magnetization may be acquired during a measurement run in an MPMS, which takes typically an hour, but not in low-field magnetic susceptibility measured in an alternating magnetic field of $465 \mathrm{~Hz}$. Despite the problem of the magnetic field accuracy, in this paper we adopt the magnetic parameters measured with the MPMS, because it provides 
Table 2. Summary of the magnetic properties of the soil samples.

\begin{tabular}{ccccc}
\hline Sample & $\begin{array}{c}\text { Magnetization }\left(12^{\circ} \mathrm{C}\right) \\
\left(10^{-3} \mathrm{~A} \mathrm{~m}^{2} / \mathrm{kg}\right)\end{array}$ & $\begin{array}{c}\text { Temperature dependence } \\
\left(10^{-6} \mathrm{~A} \mathrm{~m}^{2} / \mathrm{kg}^{\circ} \mathrm{C}\right)\end{array}$ & $\begin{array}{c}\text { Low-field magnetic susceptibility } \\
\left(10^{-5} \mathrm{SI}\right)\end{array}$ & $\begin{array}{c}\text { Bulk density } \\
\left(10^{3} \mathrm{~kg} / \mathrm{m}^{3}\right)\end{array}$ \\
\hline M1 & $1.378 \pm 0.002$ & $-3.057 \pm 0.186$ & 768 & 1.09 \\
M2 & $1.455 \pm 0.006$ & $-13.965 \pm 0.737$ & 721 & 1.10 \\
M3 & $0.747 \pm 0.000$ & $-1.737 \pm 0.041$ & 569 & 1.13 \\
M4 & $1.195 \pm 0.003$ & $-2.932 \pm 0.374$ & 661 & 1.25 \\
M5 & $4.294 \pm 0.098$ & $-56.646 \pm 11.940$ & & 1.48 \\
\hline K1 & $0.057 \pm 0.000$ & $-0.476 \pm 0.015$ & 39.9 & 1.63 \\
K2 & $0.115 \pm 0.000$ & $-0.701 \pm 0.023$ & 83.4 & 1.14 \\
K3 & $1.579 \pm 0.002$ & $-7.807 \pm 0.296$ & 665 & 1.11 \\
K4 & $1.628 \pm 0.001$ & $-4.371 \pm 0.084$ & 220 & 0.87 \\
\hline Y1 & $0.639 \pm 0.000$ & $-0.570 \pm 0.057$ & 216 & 1.01 \\
Y2 & $0.840 \pm 0.000$ & $-0.329 \pm 0.059$ & 482 & 0.91 \\
Y3 & $0.621 \pm 0.001$ & $-1.384 \pm 0.099$ & 689 & 0.98 \\
Y4 & $0.885 \pm 0.000$ & $-0.900 \pm 0.039$ & & \\
\hline
\end{tabular}

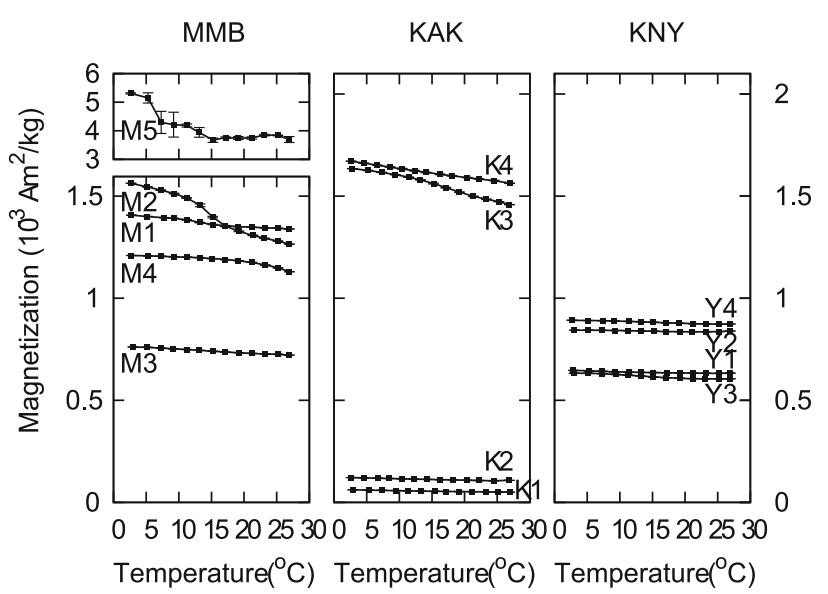

Fig. 4. Variation in magnetization during heating each sample from $2{ }^{\circ} \mathrm{C}$ to $27^{\circ} \mathrm{C}$ in a magnetic field of $0.05 \mathrm{mT}$. Numbers correspond to sample numbers shown in Table 1.

the best temperature control within the ground temperature range.

\section{Determination of Geomagnetic Annual Varia- tions}

We determined geomagnetic annual variations based on the annual variations of the ground temperature and the measured values of magnetization and its temperature dependence. Samples from MMB showed the largest temperature dependence of magnetization of the three observatories, illustrating that an annual variation exists at MMB. We determined the geomagnetic annual variations around the second variation measurement house and the absolute measurement house of MMB (96FM and ABS in Fig. 3), where absolute measurements are performed and a fluxgate magnetometer is installed, respectively.

The ground temperature measured at MMB between May 2004 and January 2005 (Nishimura et al., 2012; Fig. 5(b)) indicates that an annual temperature variation is dominant in the ground temperature variation below a depth of $0.1 \mathrm{~m}$. Assuming a simple one-dimensional thermal diffusion, the
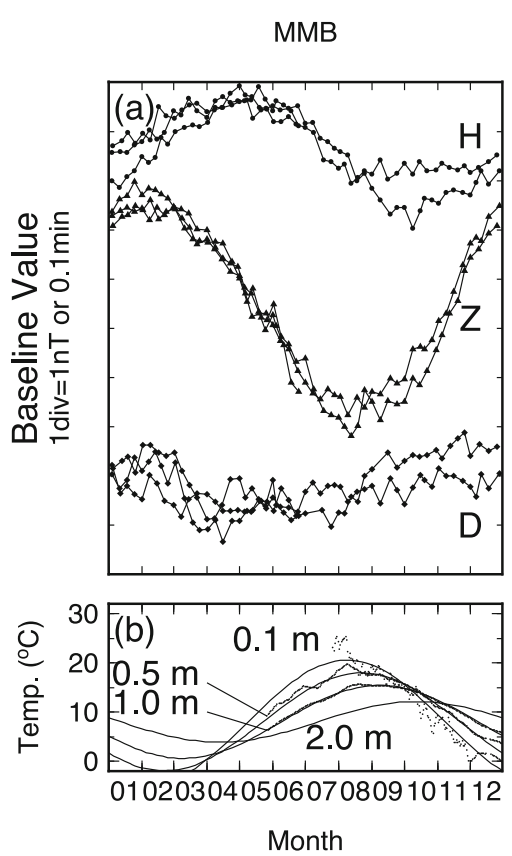

Fig. 5. (a) The annual variation in baseline values of the fluxgate magnetometer at the Memambetsu Observatory from January 2008 to June 2010. (b) Observed ground temperature at Memambetsu Observatory in 2004 (solid circles; Nishimura et al., 2012) and the determined annual variation in ground temperature (lines).

ground temperature variation $\Delta T(z, t)$ is expressed as:

$$
\Delta T(z, t)=\Delta T_{0} \exp (-n z) \cos (\omega t-n z)
$$

where $\Delta T_{0}$ is the temperature variation amplitude at the surface, $\omega=2 \pi \mathrm{yr}^{-1}$ is the angular frequency, and $n=$ $\sqrt{\omega / 2 \kappa}$ where $\kappa$ is the thermal diffusivity. According to the ground temperature record, we determined these parameters as $\Delta T_{0}=13.3^{\circ} \mathrm{C}$ and $n=0.585 \mathrm{~m}^{-1}$ (Fig. 5(b)).

We considered a strongly-magnetized rectangular body, with dimensions of $20 \mathrm{~m} \times 20 \mathrm{~m} \times 1 \mathrm{~m}$, buried within a horizontal 2-layer model (Fig. 6) as a model of the distribution of soil magnetization, for the following reasons. The depth of the magnetic anomaly body is set to $1-2 \mathrm{~m}$, because the annual variation of the $H$ and $Z$ components of 


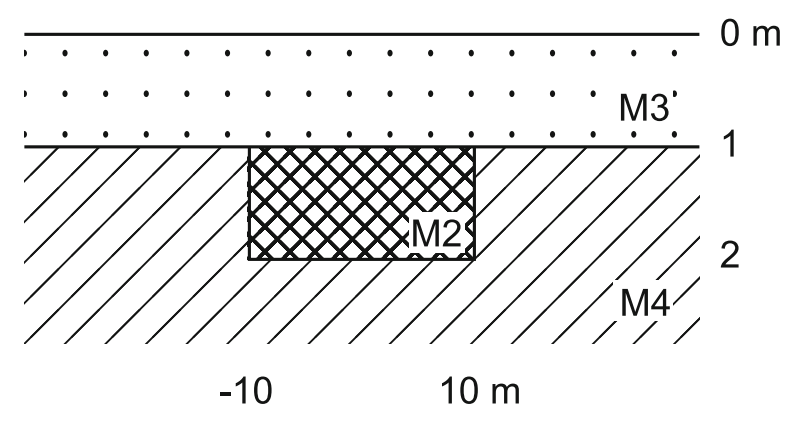

Fig. 6. A model of the magnetization distribution in the soil. We adopted the magnetization, and its temperature dependence, of the samples M2, M3 and M4 (Table 1) as those within each block.
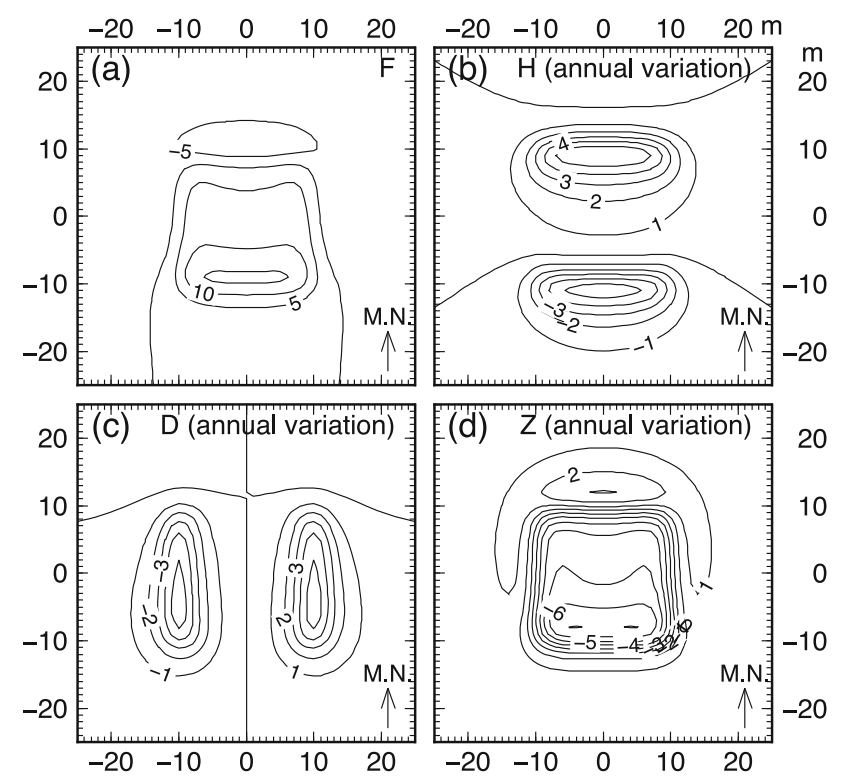

Fig. 7. The calculated distribution of the magnetic field and its annual variation. (a) Magnetic total intensity. (b)-(d) Amplitudes of annual variations for $H, D$ and $Z$ components, respectively. Positive and negative signs in (b)-(d) indicate an increase and decrease, respectively, during high ground-temperature periods.

the baseline values for the fluxgate magnetometer at MMB is in phase with the ground temperature at depths of 1-2 m (Fig. 5). The spatial distribution of the total geomagnetic intensity at MMB (Nishimura et al., 2012) shows an area with a positive geomagnetic intensity up to $\sim 10 \mathrm{nT}$, with dimensions of $\sim 20 \mathrm{~m}$, near the second variation measurement house ("96F" in figure 6 of Nishimura et al., 2012), and an area with a negative geomagnetic intensity surrounding it on the north, east and west sides, suggesting a stronglymagnetized body beneath it, in addition to a northwarddecreasing trend. Strong magnetization values of samples from the second variation measurement house (samples M1 and M2) support the existence of a strongly magnetized body. We adopt magnetization and its temperature dependence of sample M2 as those of the strongly magnetized body, and those of M3 and M4 as those of two horizontal layers (Fig. 6). The direction of magnetization in each layer is assumed to be parallel with the geomagnetic field direction.

We calculated the spatial distribution of total geomag- netic intensity and the annual variations for $H, D$ and $Z$ components at a height of $2 \mathrm{~m}$ yielded by the assumed soil magnetic structure (Fig. 7). The phase of the annual variation of all components are focused either between 48-50 degrees, or between 228-230 degrees, which are in phase and antiphase to the ground temperature at a depth of 1.43$1.49 \mathrm{~m}$, and are shown as positive and negative amplitudes, respectively. As for the total intensity, an area with a positive magnetic anomaly up to $\sim 15 \mathrm{nT}$ is located above the strongly-magnetized body, while a region with a negative magnetic anomaly surrounds it on the north, east and west sides. For the annual variations of the $H$ and $D$ components, two regions of opposite phase, with maximum amplitudes of $\sim 5 \mathrm{nT}$, appeared above the edge of the stronglymagnetized body. The annual variations of $H$ and $D$ are in phase with the ground temperature variations above the northern and eastern edges of the strong magnetization, respectively. The annual variation of $Z$ has a maximum amplitude of $\sim 7 \mathrm{nT}$, and shows a similar spatial distribution to that of the total intensity.

\section{Discussion}

The temperature dependence of magnetization is largest for the MMB samples, and smallest for the KNY samples. It is consistent with the amplitude of the annual variations, in the baseline values of the fluxgate magnetometers, which was largest at MMB and smallest at KNY. The small temperature dependence of magnetization in the KNY samples can also explain the insignificant annual fluctuations in the spatial distribution of the total geomagnetic intensity reported by Yamazaki et al. (2012).

The positive magnetic anomaly up to $\sim 15 \mathrm{nT}$ in the calculated spatial distribution of the total geomagnetic intensity (Fig. 7(a)) is similar to the local features around the second variation measurement house described above.

The ranges of the calculated annual variation amplitudes for each component are comparable to the observed annual variation amplitudes of the baseline values. At MMB, both $H$ and $Z$ components show high baseline values, (i.e. low observed values of the fluxgate magnetometer) during summer and autumn when the ground temperature is high. The observed annual variations of both $H$ and $Z$ in antiphase to the ground temperature can be explained by a calculation locating a strongly-magnetized body beneath the north of the fluxgate magnetometer. The calculated phase of the geomagnetic annual variation is common to the $H, Z$ and $D$ components, and consistent with the depth of the strong magnetization. The different phase of the observed annual variations of $H$ and $Z$ may be explained by multiple magnetization anomalies at different depths. The calculated annual variation of $D$ reaches up to $\sim 5 \mathrm{nT}$ above the eastern and western edges of the strongly-magnetized body, but is smaller than $1 \mathrm{nT}$ between them. The insignificant observed annual variation of $D$ can be explained by assuming that the fluxgate magnetometer is located above the central region of strong magnetization.

\section{Conclusion}

The annual variations in the geomagnetic field caused by changes in soil magnetization in response to temperature 
changes was determined from measuring the magnetization, and its temperature dependence, of soil samples collected from three JMA magnetic observatories. The amplitude of the determined annual variations at the MMB observatory was up to $\sim 7 \mathrm{nT}$, which corresponded approximately with the observed annual variations in the baseline values of the fluxgate magnetometer.

Acknowledgments. We thank Toshitsugu Yamazaki and Hirokuni Oda for the use of their facilities. We also thank two anonymous reviewers for their constructive comments.

\section{References}

Kawamura, N., H. Oda, K. Ikehara, T. Yamazaki, K. Shioi, S. Taga, S. Hatakeyama, and M. Torii, Diagenetic effect on magnetic properties of marine core sediments from the southern Okhotsk Sea, Earth Planets Space, 59, 83-93, 2007.

Machida, H. and F. Arai, Atlas of Volcanic Ash, 276 pp., Tokyo University Press, Tokyo, Japan, 2003 (in Japanese).

Nishimura, M., S. Arita, T. Moriyama, M. Hashimoto, M. Sugawara, N. Ishida, and $\mathrm{H}$. Hasegawa, Survey of annual fluctuations in total geomagnetic intensity observations, Tech. Rep. Kakioka Magn. Obs., Suppl. 10,
$13-17,2012$.

Ogawa, T. and S. Koyama, Temporal variation of the magnetic anomaly in the absolute measurement house of Yatsugatake Geoelectromagnetic Observatory, Proc. Conductivity Anomaly Symposium, 74-77, 2009 (in Japanese).

Oowada, T., T. Tokumoto, Y. Yamada, M. Ozima, N. Kumasaka, M. Yokoyama, M. Sugawara, K. Koike, and Y. Shimizu, Introduction to our new system: Magnetometer for wide frequency range, Tech. Rep. Kakioka Magn. Obs., Suppl. 1, 1-14, 2003.

Oyama, M. and H. Takehara, Revised Standard Soil Color Charts, 72 pp., Fujihira Industry Co., Tokyo, Japan, 1967.

Utada, H., M. Neki, and T. Kagiyama, A study of annual variations in the geomagnetic total intensity with special attention to detecting volcanomagnetic signals, Earth Planets Space, 52, 91-103, 2000.

Yamazaki, A., N. Shigeno, T. Yamamoto, Y. Kumagai, and N. Itoh, Magnetic anomaly caused by soil excavation and its long-term stability: Case study of a magnetic anomaly in the absolute observation house at Kanoya Magnetic Observatory, Tech. Rep. Kakioka Magn. Obs., Suppl. 10, 1-12, 2012 .

T. Mishima (e-mail: mishima@sci.osaka-cu.ac.jp), T. Owada, T. Moriyama, N. Ishida, K. Takahashi, S. Nagamachi, Y. Yoshitake, Y. Minamoto, F. Muromatsu, and S. Toyodome 